\title{
COMENTARIO
}

\section{Conservación de la diversidad genética en el Perú: desafíos en la implementación del régimen de acceso a recursos genéticos y distribución de beneficios}

\section{Conserving the genetic diversity in Peru: challenges in the implementation of an access and benefit-sharing regime}

\section{Luciana Carla Silvestri}

Cátedra UNESCO de Territorio y Medio Ambiente - Universidad Rey Juan Carlos, España.

Dirección postal: c/ Tulipán s/n- Móstoles 28933 (España). Edificio Departamental II. Oficina 241

Email Luciana Silvestri: silvestriluciana@yahoo.com.ar

\begin{abstract}
Resumen
Esta contribución debate los grandes retos que enfrenta Perú en la implementación nacional del régimen de acceso a recursos genéticos y distribución de beneficios, tema conocido por las siglas "ABS" del inglés Access and Benefit-Sharing. El ABS es un mecanismo que incentiva la conservación de la diversidad genética mediante la distribución justa y equitativa de los beneficios que se deriven de la utilización de recursos genéticos entre el país que los provee y el usuario de éstos. Perú afronta cuatro desafíos en relación con el tema: (1) su procedimiento de acceso a recursos genéticos es complejo y burocrático, desalentándose así la negociación de contratos sobre ABS. (2) el rol de la "institución nacional de apoyo" ha sido desvirtuado, exacerbándose su función de mero controlador; con ello se han perdido valiosas colaboraciones científicas a favor de las instituciones científicas locales. (3) la legislación peruana sobre ABS posee efectos retroactivos, infringiendo el principio de seguridad jurídica que debe reinar en toda relación contractual. (4) si bien se han previsto medidas de cumplimiento y un punto de verificación de acuerdo a lo establecido por el Protocolo de Nagoya, no se establece un control sobre la legalidad del acceso a recursos genéticos y conocimiento tradicional asociado al uso de éstos cuando ellos provengan de cualquier otro país que no sea el Perú. Al finalizar el artículo se proponen algunas mejoras a realizar a fin de afrontar los desafíos encontrados en el estudio.
\end{abstract}

Palabras claves: ABS; biodiversidad; Protocolo de Nagoya; biotecnología; Perú.

\section{Abstract}

This essay intends to debate the major challenges that Peru faces as it implements a national regime on Access to genetic resources and Benefit-Sharing (ABS). The ABS encourages the conservation of genetic diversity by means of the fair and equitable distribution of the benefits arisen from the utilization of genetic resources. Distribution is to take place between the providing country and the user of the resources. Peru features four main weaknesses in relation to ABS: (1) Firstly; its national legislation on the topic sets a bureaucratic and complex procedure that hinders the negotiation of fructiferous ABS contracts. (2) The role of the "national scientific partner" has been distorted and true opportunities for scientific cooperation are missed. (3) Peruvian legislation on ABS establishes retroactive effects infringing the principle of legal certainty. (4) Even though the national legal framework establishes a set of compliance measures and a checkpoint to verify the legal access of genetic resources in accordance to the Nagoya Protocol, these measures do not cover the control of genetic resources and traditional knowledge associated to their utilization from countries other than Peru. Improvements to face the challenges encountered are offered at the end of the article.

Key words: ABS; biodiversity; Nagoya Protocol; biotechnology; Peru.

\section{Citación:}

Silvestri L.C. 2016. Conservación de la diversidad genética en el Perú: desafíos en la implementación del régimen de acceso a recursos genéticos y distribución de beneficios. Revista peruana de biología 23(1): 073 - 079 (Abril 2016). doi: http://dx.doi.org/10.15381/rpb.v23i1.XXXX

Presentado: $\quad$ 02/12/2015

Aceptado: $\quad 16 / 04 / 2016$

Publicado online: $28 / 05 / 2016$
Información sobre los autores:

La autora declara que no incurre en conflictos de intereses. 


\section{Introducción}

Perú goza de una diversidad de ecosistemas, especies y recursos genéticos exuberante, encontrándose entre los diez países más biodiversos del mundo (MINAM 2010a). El 10\% de todas las especies de flora identificadas a nivel mundial se encuentran presentes en su territorio (Pastor Soplín 2004), y con menos del $1 \%$ de la superficie global terrestre, alberga 20375 especies de plantas vasculares (MINAM 2010b); es decir, el $7.5 \%$ del total registrado globalmente. De ellas, al menos 5509 son endémicas (León et al. 2006). En relación con la fauna, si bien se encuentra menos estudiada, el país también presenta una riqueza extraordinaria: Perú se halla en cuarto lugar a nivel mundial en relación con el número de especies de anfibios -538- (Aguilar et al. 2010), segundo si se considera las especies de aves -1835-(Plenge 2010) y quinto si se trata de mamíferos -508 especies- (Pacheco et al. 2009). Tal diversidad biológica convierte al Perú en un proveedor innato de recursos genéticos altamente valiosos para el sector biotecnológico; habiéndose estimado en este sentido, que cerca de 400 especies de plantas nativas son potencialmente utilizables por la industria farmacológica (Kámiche Zegarra 2010).

Desde esta perspectiva, Perú podría beneficiarse si implementase a nivel nacional un régimen eficiente y efectivo de acceso a recursos genéticos y distribución de beneficios, mecanismo conocido como "ABS" por sus siglas en inglés de Access and Benefit-Sharing. El ABS es capaz de lograr dos objetivos fundamentales. En primer lugar, desacelerar la pérdida de biodiversidad -y específicamente de la diversidad genética- mediante la distribución de los beneficios que se deriven de la utilización de recursos genéticos con los países que los proveen, quienes generalmente son ricos en biodiversidad pero pobres en recursos financieros (Godt 2009). En segundo término, compensar a las comunidades indígenas y locales por el uso de su conocimiento tradicional en relación con la biodiversidad; ya que si bien dicha información ha sido utilizada frecuentemente para el avance de la ciencia, ellas nunca han sido retribuidas (Aguilar 2001).

Estas oportunidades, aunque reales, no son fáciles de lograr, ya que la implementación nacional de un régimen de $\mathrm{ABS}$ supone grandes dificultades. Éstas incluyen la desvinculación de las políticas de ABS con la realidad social, económica, cultural, política, científica e institucional de un país, la escasa capacidad humana e institucional para conceder acceso y negociar contratos sobre reparto de beneficios y la falta de mecanismos institucionales para coaccionar el cumplimiento de la legislación (Lewis-Lettington et al. 2006), la existencia de lagunas legales en temas esenciales a la hora de regular el ABS (Chishakwe \& Young 2003) y el enquistamiento en posiciones irreales, generalmente sólo enfocadas en beneficios monetarios, en detrimento de otras oportunidades más realistas y alcanzables al corto plazo como la cooperación en investigación científica y la transferencia de tecnología (Glowka 2000).

Esta investigación, oportuna de cara a la reciente ratificación del Protocolo de Nagoya por parte de Perú ocurrida el 8 de julio de 2014, busca analizar las debilidades que presenta el marco normativo de acceso a recursos genéticos y distribución de beneficios del país. A través de ella se echará luz sobre el novedoso mecanismo de ABS dispuesto por el Protocolo de Nagoya, las debilidades que muestra el cuadro normativo peruano sobre $\mathrm{ABS}$ y las mejoras a introducir en el régimen actual para enfrentar los retos hallados.

\section{Metodología}

Luego de analizar histórica y jurídicamente las normas internacionales donde se sustenta el sistema de ABS -Convenio sobre la Diversidad Biológica y Protocolo de Nagoya- se investigó el régimen peruano de $\mathrm{ABS}$ mediante el método jurídico, siguiéndose en particular un enfoque descriptivo, comparativo y propositivo. Si bien la legislación peruana se halla condicionada por la Decisión 391 de la Comunidad Andina que establece el régimen común sobre acceso a los recursos genéticos para los países integrantes del Grupo, esta investigación trata principalmente, aunque no en exclusividad, sobre las normas de origen puramente peruano. Ello se debe a la existencia de abundantes y completos estudios que ya analizan la norma andina, como por ejemplo los trabajos de Caillaux Zazzali et al. (1999), Rosell (1997) y Ruiz Müller (2008), y a la inexistencia de estudios post-Protocolo de Nagoya sobre el régimen peruano de ABS. A fin de completar el análisis documental y jurídico se realizaron entrevistas individuales no estructuradas con algunos de los principales actores del ABS del país. La legislación peruana fue analizada mediante una matriz de análisis estratégico desarrollada en Silvestri (2014), la misma permite evaluar las capacidades normativas de cualquier país para implementar un sistema efectivo de ABS y satisfacer al mismo tiempo las obligaciones establecidas en el Protocolo de Nagoya.

El marco normativo internacional: Convenio sobre la Diversidad Biológica y Protocolo de Nagoya.- El Convenio sobre la Diversidad Biológica (CDB) es un acuerdo casi global, con la notoria ausencia de Estados Unidos, que trata de conjugar los diferentes, y a veces divergentes, intereses de las Partes que lo negociaron: mientras que los países desarrollados priorizaron la conservación de la biodiversidad (Glowka et al. 1994), sus pares en vías de desarrollo privilegiaron el desarrollo humano, el uso sostenible de la biodiversidad, la transferencia de tecnología, el financiamiento para la conservación y el reparto de beneficios provenientes de la utilización de recursos genéticos (Cabrera Medaglia 1994, Raustiala \& Victor 1996). Surgido como un acuerdo que busca satisfacer a todas las Partes se caracteriza, al menos en los que respecta al ABS, por sus claras implicaciones redistributivas (Raustiala 1997).

El CDB, en vigor desde el 29 de diciembre de 1993, reiteró el reconocimiento del principio de soberanía de los Estados sobre los recursos naturales que se encuentran bajo su jurisdicción (art. 3). Además dispuso la potestad de las Partes para regular el acceso a sus recursos genéticos (art. 15.1), estableciendo que si éstas deciden reglamentarlo, quien desee entonces acceder a ellos deberá contar con el consentimiento fundamentado previo de dicha Parte (art. 15.5) y acordará las condiciones del acceso y uso de recursos genéticos con ella (art. 15.4). El CDB también protege el conocimiento tradicional asociado a la utilización de la biodiversidad en posesión de comunidades indígenas y locales, obligando a las Partes a fomentar el reparto equitativo de los beneficios derivados de la utilización de este conocimiento con las comunidades que lo proveen (art. $8 \mathrm{j}$ ).

A pesar de las disposiciones del CDB, la falta de medidas de control en el país del usuario de recursos genéticos que sirvieran para verificar que éstos hubieran sido accedidos de acuerdo con la legislación del país proveedor, socavaba todo el sistema. Como consecuencia de ello, un grupo de países mega-diversos, constituido por Brasil, China, Costa Rica, Colombia, Ecuador, 
India, Indonesia, Kenia, México, Perú, Sudáfrica y Venezuela, decidió buscar una nueva instancia negociadora sobre ABS a fin de resolver la falta de medidas de control (Lago Candeira 2007). El interés de dichos países que fue plasmado primero en la Declaración de Cancún de Países Mega-Diversos Afines y recogido posteriormente en el Plan de Aplicación de las Decisiones de la Cumbre Mundial sobre el Desarrollo Sostenible (punto 44 o) concretado en 2002 en Johannesburgo, Sudáfrica, dio origen al Protocolo de Nagoya.

El Protocolo de Nagoya entró en vigor el 12 de octubre de 2014. El acuerdo dispone que el acceso a recursos genéticos se deba producir únicamente cuando medie consentimiento fundamentado previo del país proveedor (art. 6.1) y una distribución justa y equitativa de beneficios entre el solicitante de acceso y el proveedor de los recursos, concretada en condiciones mutuamente acordadas (art. 5.1). Entre estos beneficios, los cuales pueden ser monetarios y no monetarios, el Protocolo privilegia el acceso y transferencia de tecnología a favor del país proveedor y la colaboración y cooperación científica con él (art. 23). Asimismo, el Protocolo de Nagoya regula el acceso al conocimiento tradicional asociado a la utilización de recursos genéticos que se encuentra en posesión de comunidades indígenas y/o locales; disponiendo que tal acceso se realice únicamente cuando medie consentimiento fundamentado previo de las comunidades y con la participación de éstas en los beneficios que del uso del conocimiento pudieran derivarse (art. 7).

Finalmente, el Protocolo establece las famosas medidas de cumplimiento. En tal sentido, el Protocolo obliga a adoptar medidas legislativas, administrativas o de política apropiadas, eficaces y proporcionales para asegurar que los recursos genéticos utilizados dentro de la propia jurisdicción hayan sido accedidos de conformidad con el consentimiento fundamentado previo del país proveedor y que se han establecido con él condiciones mutuamente acordadas de acuerdo con su legislación nacional (art. 15.1). Además se dispone la misma obligación con respecto al conocimiento tradicional asociado a la utilización de recursos genéticos (art. 16.1). Como corolario de lo anterior, las Partes deben además adoptar medidas para abordar situaciones de incumplimiento con respecto a las medidas de cumplimiento anteriormente mencionadas (art. 15.2 para recursos genéticos) y (art. 16.2 para conocimiento tradicional).

Si bien las medidas de cumplimiento no han sido especificadas, ellas deben ofrecer un control efectivo y eficiente sobre el status legal de los recursos genéticos y/o conocimiento tradicional que se utilizan en la propia jurisdicción ya que de otra manera todo el régimen articulado por el Protocolo sería inútil. Una medida de cumplimiento que obligatoriamente debe establecerse es la designación de un "punto de verificación" encargado de controlar la legalidad del acceso a los recursos genéticos (art. 17.1 (a)). No se aclara qué organismo deberá actuar como punto de verificación pero éste podría ser la oficina de patentes, el organismo nacional encargado de financiar investigación sobre recursos genéticos u otro que la Parte desee y cumpla con los requisitos enunciados en el Protocolo (art. 17.1 (a) (iv)).

También es novedosa la introducción del "permiso" (art. 6.3 (e)) o "certificado de cumplimiento" (art. 17.2), el cual acredita el cumplimiento del consentimiento fundamentado previo y el establecimiento de condiciones mutuamente acordadas con el país proveedor de los recursos genéticos. El certificado será exi- gible más tarde en la jurisdicción del propio usuario de recursos genéticos en el momento en que él pase por alguno de los puntos de verificación establecidos (art. 17.1 (a) (i) (ii) (iii)). De esta manera se demostrará que efectivamente se ha cumplido con la legislación nacional del país proveedor de recursos genéticos al acceder a ellos.

\section{Resultados y discusión Desafíos que presenta la legislación peruana}

Procedimiento de acceso a recursos genéticos.- El procedimiento de acceso a recursos genéticos de Perú se encuentra principalmente regulado mediante la Decisión Andina 391 y el Decreto Supremo N 003-2009-MINAM que aprueba el Reglamento de Acceso a Recursos Genéticos, la principal norma de ABS del país. Asimismo, los novedosos Decreto Supremo $\mathrm{N}^{\circ}$ 018-2015-MINAGRI y Decreto Supremo Nº19-2015-MINAGRI que aprueban respectivamente el Reglamento para la Gestión Forestal y el Reglamento para la Gestión de Fauna Silvestre han introducido disposiciones de importancia con respecto al tema.

El Decreto $N^{\circ}$ 003-2009-MINAM establece un procedimiento de acceso extremadamente complejo y burocrático. Dicha falta es achacable prioritariamente a la Decisión, que es donde se definen los elementos estructurales del trámite, y a la cual el Decreto sólo completa con detalles poco importantes en este aspecto. En ella por ejemplo, se establecen una gran variedad de requisitos para iniciar el procedimiento, numerosas instancias a consultar así como largos plazos administrativos. La legislación peruana sin embargo, también muestra una cierta burocratización de los procedimientos en lo que particularmente de ella depende. Así por ejemplo, establece que quien desee acceder a recursos genéticos de la flora o fauna silvestre o sus derivados deberá contar necesariamente con un título habilitante para el aprovechamiento de los recursos biológicos que los contengan (art. 146 del Decreto Supremo No 018-2015-MINAGRI y art. 126 del Decreto Supremo Nº19-2015-MINAGRI). Si bien ambas solicitudes pueden tramitarse paralelamente, se estima introducen mayor complejidad y tiempo de espera al trámite de acceso a recursos genéticos.

En segundo término, las gestiones relacionadas con el ABS se ven obscurecidas por el desconocimiento de la norma y la falta de capacidades para negociar contratos de acceso que aducen los funcionarios competentes en la materia, debilidades que impactan negativamente en la eficacia del proceso pues tornan reticentes al personal administrativo a actuar por miedo a equivocarse. Terceramente, la falta de "Textos Únicos de Procedimientos Administrativos (TUPAS)", una especie de listado abreviado que tiene cada administración del Estado sobre los requisitos necesarios para dar trámite y gestionar cada uno de los probables procedimientos administrativos que le pudieran competer, lleva a los funcionarios a invocar su incapacidad para actuar ante una aducida falta de norma. Es necesario aclarar que en ningún caso los TUPAS constituyen una norma o agregan o eliminan requisitos, instancias o plazos que la ley haya establecido, sino que simplemente son un check list. Sin embargo, en la práctica administrativa del Perú, el temor de los funcionarios los lleva a sostener que sin TUPA no se puede proceder. Y, como aún no hay TUPAS para solicitar acceso a recursos genéticos, las solicitudes de acceso van de una oficina a otra con la esperanza de que alguien decida qué hacer. Con respecto a este 
punto, es menester aclarar que Perú perdió recientemente una valiosa oportunidad para contar con un TUPA para el acceso a recursos genéticos; ya que si bien en septiembre de 2015 aprobó un nuevo Texto Único de Procedimientos Administrativos del Ministerio de Agricultura y Riego a través del Decreto Supremo Nº16-2015-MINAGRI, no incluyó en esa oportunidad el trámite de acceso a recursos genéticos entre los varios que sí previó.

Por último, hasta recientemente, la inexistencia de procedimientos diferenciados para el acceso a recursos genéticos con fines puramente científicos obstaculizaba también la investigación no comercial, al obligar a los científicos a tramitar su solicitud por el mismo procedimiento y cumpliendo los mismos requisitos que cuando la investigación perseguía fines comerciales. Afortunadamente, los novedosos Decretos $N^{\circ}$ 018-2015-MINAGRI (art. 154) y $\mathrm{N}^{\circ}$ 019-2015-MINAGRI (art. 34.5) revierten esta situación, al prever que las actividades de investigación básica taxonómica de flora y fauna silvestre, relacionadas con estudios moleculares con fines taxonómicos, sistemáticos, fitogeográficos, biogeográficos, evolutivos y de genética de la conservación, entre otras investigaciones sin fines comerciales, sean aprobadas mediante autorizaciones de investigación científica y no requieran por lo tanto, de un contrato de acceso a recursos genéticos. Dicha diferenciación de procedimientos es, por otra parte, obligatoria de acuerdo a lo establecido en el Protocolo de Nagoya (art. 8.a).

Como resultado de todo ello hasta mayo de 2013 no se había suscripto ningún contrato de acceso a recursos genéticos en condiciones in situ y con fines comerciales en el país. Tampoco se había firmado contrato marco alguno que avalara el acceso frecuente a recursos genéticos con fines puramente de investigación científica por parte de universidades o instituciones científicas. Ello lleva a concluir que probablemente los recursos genéticos del país están siendo accedidos y utilizados por mecanismos diferentes a los previstos por el Decreto $\mathrm{N}^{\circ}$ 003-2009-MINAM; por ejemplo, a través del uso de acuerdos de transferencia de material (ATM), los cuales sólo pueden ser utilizados cuando el objeto de la trasferencia sea la investigación con fines puramente científicos (art. 29), o simplemente están siendo accedidos de forma ilegal.

Por otro lado, a la legislación peruana le resta por incorporar varias de las novedades introducidas por el Protocolo de Nagoya en relación con el acceso. Por ejemplo falta que el Decreto prevea la instancia final del procedimiento administrativo por la cual se emita un "permiso" que certifique el cumplimiento del marco nacional de acceso en relación a los recursos genéticos según lo exigido por el Protocolo de Nagoya (art. 6.3.e). La actual "resolución de acceso" de la normativa peruana, podría equipararse al "permiso" exigido por el Protocolo siempre y cuando, se inserte en ella el mismo contenido que establece para el permiso el Protocolo (arts. 6.3.e y 17.4). Asimismo habría que ajustar su formato al que finalmente establezca la Secretaría del CDB o las Partes del Protocolo. Por otro lado, falta que el Decreto establezca consideraciones especiales para los recursos genéticos útiles para la alimentación y la agricultura, que cumplen un rol especial para la seguridad alimentaria (art. 8.c) y que están excluidos del Tratado Internacional sobre Recursos Fitogenéticos para la Alimentación y la Agricultura, del cual Perú es Parte. Por último, no se han observado reglas especiales en la normativa peruana que posibiliten el acceso expedito a recursos genéticos necesarios en caso de emergencia que cree amenazas o daños para la salud humana, animal o vegetal a cambio de una participación justa y equitativa y expeditiva en los beneficios que se deriven del uso de dichos recursos genéticos, incluido el acceso a tratamientos asequibles para los necesitados (art. 8.b).

Rol de la institución nacional de apoyo.- El rol de la "institución nacional de apoyo”, pensada en la Decisión 391 como una contraparte nacional, dedicada a la investigación científica o técnica, que acompañe al solicitante de acceso a recursos genéticos y participe junto con él en las actividades de acceso (art. 1), ha sido desvirtuado. En la actualidad tanto el Decreto Supremo $\mathrm{N}^{\circ}$ 003-2009-MINAM que la regula en sus artículos 18 y 19 , como la práctica administrativa del país han impreso a la institución un marcado sesgo de control. Con ello la función real que ejerce la institución nacional de apoyo se circunscribe a verificar que las actividades de acceso e investigación que el solicitante de recursos genéticos lleva a cabo se ajustan a la resolución que autoriza el acceso. Este giro ha llevado a pensar que la exigencia de hacerse acompañar por una institución nacional de apoyo es una forma solapada de seleccionar quién será el "espía" que controle el proyecto; repercutiendo dicha percepción negativa, en el efectivo comienzo de varias iniciativas de bioprospección. Se estima por lo tanto que debido a este cambio sustancial de funciones se han perdido valiosas oportunidades para la participación del sector científico peruano en la investigación biotecnológica, o al menos en alguna de sus etapas.

Retroactividad.- El cuadro normativo de ABS de Perú posee claros efectos retroactivos. Con ello se vulnera el principio de seguridad jurídica que busca crear certidumbre sobre los derechos y obligaciones que corresponden a un determinado sujeto y que debe, dada la universalidad del principio, primar en todo ordenamiento normativo. Disposiciones del Decreto $\mathrm{N}^{\circ}$ 003-2009-MINAM tales como las que obligan a quien detente recursos genéticos, productos derivados o componente intangible originarios de Perú a regularizar el acceso en el plazo de un año desde la entrada en vigencia de la legislación bajo apercibimiento de aplicar diversas sanciones (disposición transitoria segunda), o a ajustar a la reglamentación vigente los contratos o convenios que se hubieren suscripto con terceros sobre recursos genéticos, sus productos derivados o los recursos biológicos que los contengan bajo pena de no poder acceder nuevamente a recursos genéticos (disposición transitoria tercera), o facultan al Estado para ejercer acción reivindicatoria y para cobrar la indemnización correspondiente con respecto a recursos genéticos peruanos (disposición transitoria sexta), claramente amplían las consecuencias jurídicas establecidas por la reglamentación a hechos (accesos y acuerdos) acaecidos con anterioridad a su entrada en vigencia. En cualquier caso resulta mencionable que el impacto real de estas disposiciones ha sido nulo ya que no se ha producido el pretendido "saneamiento" o "regularización".

Por otro lado, parece claro que las disposiciones bajo comentario, que hallan asidero en la Decisión 391, pueden entrar en conflicto con el ámbito temporal del CDB y del Protocolo de Nagoya; puesto que ninguno de los dos instrumentos internacionales indica retroactividad alguna. En este sentido, el problema que Perú podría enfrentar al reclamar a terceros países Parte del CDB o del Protocolo de Nagoya -basado en un acceso producido con anterioridad a la adopción del Decreto Supremo $\mathrm{N}^{\circ}$ 003-2009-MINAM y fundándose en la retroactividad de éste-, es que muy probablemente estos países no atenderían tal reque- 
rimiento al entender que él se apoya en una norma unilateral y no en acuerdos multilaterales. Lamentablemente, el nuevo proyecto de reglamento de ABS que se encuentra bajo consulta pública (Resolución Ministerial N²33-2013-MINAM) repite estas disposiciones de carácter retroactivo.

Medidas de cumplimiento y punto de verificación.Perú había implementado a través del Decreto Supremo $N^{\circ}$ 003-2009-MINAM una serie de medidas de cumplimiento con anterioridad al Protocolo de Nagoya en coincidencia con las que éste pretende ahora que los países adopten. Una de ellas es el "Mecanismo Nacional de Supervisión y Seguimiento Integrado de los Recursos Genéticos", cuyo objetivo es asegurar que se haya otorgado consentimiento fundamentado previo y se hayan establecido condiciones mutuamente acordadas al acceder a recursos genéticos (art. 38). El mecanismo propone un intercambio de información entre diferentes autoridades y el registro público de contratos de acceso a recursos genéticos. Otra medida de cumplimiento dispuesta por la legislación peruana incluye una serie de infracciones y sanciones entre las que destacan la suspensión o cancelación de la autorización de acceso, el decomiso del material y multas para quienes realicen actividades de acceso sin contar con la respectiva autorización o en contravención de lo establecido por el Decreto (arts. 34 y 35 ).

El Decreto Legislativo № 1075 también prevé sanciones para aquel que no presente el contrato de acceso a recursos genéticos y a conocimiento tradicional cuando se solicite una patente (art. 120 A). Ellas incluyen multas, compensación, distribución justa y equitativa de beneficios, la distribución de regalías y/o otras medidas monetarias o no monetarias, transferencia de tecnología, fortalecimiento de capacidades y autorizaciones de uso. Lamentablemente, el Decreto Legislativo en su nueva redacción (el Decreto Legislativo $\mathrm{N}^{\circ} 1075$ ha sido modificado por Ley $\mathrm{N}^{\circ} 29316$, del 13 de enero de 2009, que modifica, incorpora y regula diversas disposiciones a fin de implementar el Acuerdo de Promoción Comercial suscrito entre el Perú y los Estados Unidos de América) se ha apartado de las sanciones que prevén la Decisión 391 para la falta de presentación del contrato: no reconocimiento de derechos de propiedad intelectual, y la Decisión 486: nulidad de la patente o denegación de la misma. Estas sanciones más rigurosas, que habían sido acogidas por la legislación peruana en un primer momento, han sido sustituidas ante la necesidad de adoptar normas más benévolas a fin de lograr el acuerdo de libre comercio con Estados Unidos.

En cualquier caso, se debe reconocer que Perú sí cuenta con medidas de cumplimiento tal como las que exige el Protocolo de Nagoya y todo lo que resta por completar, de cara a estar en pleno cumplimiento con el instrumento internacional, es extender tales medidas al control de recursos genéticos y conocimientos tradicionales provenientes de cualquier país que sea Parte del Protocolo de Nagoya, y no sólo como hasta hoy, a los recursos o conocimientos provenientes del Perú.

En relación con el punto de verificación el Decreto establece que como parte del proceso de registro de derechos de propiedad intelectual correspondiente a productos que hubieren utilizado recursos genéticos o conocimientos tradicionales de los cuales el Perú es país de origen se requerirá la presentación del correspondiente contrato de acceso o en su caso del certificado (disposición complementaria quinta). Felizmente el Decreto Supremo $\mathrm{N}^{\circ}$ 003-2009-MINAM, en concordancia con la Decisión 486 de la
Comunidad Andina, extiende el control que realiza el punto de verificación al acceso y utilización del conocimiento tradicional de las comunidades indígenas y/o locales peruanas. En la Decisión 391, en cambio, el punto de verificación sólo examinaba el legal acceso y utilización de recursos genéticos y derivados.

Esta medida de la legislación peruana es muy positiva ya que con ella se cumple con lo exigido por el Protocolo de Nagoya, el cual si bien no requiere un "punto de verificación" para conocimientos tradicionales, sí manda adoptar "medidas de cumplimiento" para asegurar la legalidad y justicia en el acceso a conocimientos tradicionales asociados a recursos genéticos, siendo un punto de verificación una de las posibles medidas a establecer. Sólo restaría agregar que este punto de verificación extendiera su control a los conocimientos tradicionales de comunidades indígenas y/o locales asentadas en cualquier país Parte del Protocolo de Nagoya, y no sólo a los provenientes de comunidades del Perú, para estar en total cumplimiento con el Protocolo de Nagoya. Hasta tanto eso no ocurra, la legislación peruana presenta una cierta debilidad en la materia.

La disposición comentada se ve reforzada, al menos en relación con recursos genéticos, con otra medida que exige que cuando se solicite el otorgamiento de una patente o certificado de obtentor para productos o procesos obtenidos o desarrollados a partir de recursos genéticos de los cuales el Perú es país de origen, se debe presentar copia del correspondiente certificado de acceso y de la resolución que lo autoriza o el número de registro del contrato de acceso (disposición final primera).

Llama la atención que ambas disposiciones sólo se refieran a los “recursos genéticos de los cuales Perú es país de origen" y no lo haga, conforme a la Decisión 391, a los recursos genéticos de los Países miembros de la Comunidad Andina, ya que entre ellos se deben dar trato nacional (art. 11). Por otro lado, el Decreto sólo cumple parcialmente con lo establecido por el Protocolo de Nagoya ya que la vigilancia es sólo ejercida sobre recursos genéticos originarios del Perú y no, sobre recursos genéticos de todas las Partes del Protocolo de Nagoya. Esto es por otro lado lógico, ya que en el momento de dictarse el Decreto, el Protocolo todavía no existía. Sin embargo, para adecuarse a él, el Decreto tendría que extender el control a todos los recursos genéticos originarios de cualquier país Parte del Protocolo.

\section{Conclusiones}

Perú ha ejercido efectivamente el derecho soberano para regular el acceso a sus recursos genéticos. Su marco normativo se encuentra principalmente configurado por el Decreto Supremo $\mathrm{N}^{\circ}$ 003-2009-MINAM que reglamenta el acceso a recursos genéticos. A pesar de que el régimen es completo y detallado, el país enfrenta graves dificultades a la hora de implementar un régimen efectivo y eficiente de $\mathrm{ABS}$ a nivel nacional.

En primer lugar, el procedimiento de acceso a recursos genéticos es demasiado complejo y burocrático; como consecuencia de ello, no existen experiencias exitosas sobre ABS en el país. Amén de ello, el Decreto no incorpora las novedades del Protocolo de Nagoya en materia de acceso; por ejemplo, el permiso o consideraciones especiales para los recursos genéticos útiles para la alimentación y la agricultura no comprendidos en el Tratado Internacional sobre Recursos Fitogenéticos para la Alimentación y la Agricultura (FAO 2009). Esto último no sería del todo reprochable si al menos el proyecto de reglamento 
que se encuentra bajo tratamiento las incluyese, cuestión que lamentablemente no hace. Tal carencia supone que cualquier nuevo proyecto de reglamentación sobre $\mathrm{ABS}$ quede ya obsoleto aún antes de haber sido aprobado.

Una segunda debilidad destacable es el hecho de que el rol de la "institución nacional de apoyo" ha sido adulterado, limitándolo simplemente al mero control de las actividades que ejerce el solicitante de recursos. Con ello se ha perdido una gran oportunidad para promover el sector científico nacional el cual podría beneficiarse si se requiriese establecer con él una verdadera relación de colaboración científica.

Terceramente, la norma peruana establece efectos retroactivos restando seguridad jurídica a todas las relaciones que sobre acceso a recursos genéticos se pudieran haber establecido. Lamentablemente, el nuevo proyecto de reglamentación repite este error.

Además, si bien la normativa peruana establece medidas de cumplimiento para verificar la legalidad del acceso a recursos genéticos y conocimiento tradicional cuando éstos son peruanos y utilizados en la propia jurisdicción -medidas exigidas por el Protocolo de Nagoya-, las mismas no cubren, tal y como lo establece el Protocolo, el control de aquéllos cuando provienen de cualquier otro país que sea Parte del instrumento internacional. Igualmente, aunque Perú ha establecido un punto de verificación para controlar que el acceso a recursos genéticos se haya realizado legalmente -el cual tiene lugar durante el registro de derechos de propiedad intelectual-, el control que éste lleva a cabo sólo se limita a los recursos peruanos, y no como exige el Protocolo, a los procedentes de cualquier país Parte del mismo.

En base a lo anterior, se recomienda que Perú prevea en el nuevo proyecto de reglamentación un procedimiento más eficiente para el acceso a recursos genéticos. Igualmente, la capacitación de los funcionarios en relación con la aplicación de la legislación es crucial. También resulta de suma importancia para los intereses científicos del país recuperar la función primigenia para la que fue pensada la institución nacional de apoyo, permitiendo así que el país efectivamente se beneficie de la implementación de un mecanismo tal como el de ABS. Igualmente, es aconsejable que el nuevo proyecto de legislación elimine toda disposición que establezca efectos retroactivos sobre hechos pasados; con ello se logrará seguridad jurídica y se atraerá a potenciales investigadores a pesquisar la biodiversidad del país. Por último, sería deseable que el nuevo proyecto de reglamentación extendiera la verificación que están llamados a ejercer las medidas de control y el punto de verificación establecidos a los recursos genéticos y conocimientos tradicionales provenientes de cualquier país que sea Parte del Protocolo de Nagoya, y no sólo como en la actualidad, a los procedentes del Perú. Asimismo, debería establecerse la obligación de comunicar la información obtenida en los puntos de verificación a la Parte que otorga el consentimiento fundamentado previo y al Centro de Intercambio de Información sobre Acceso y Participación en los beneficios del Protocolo para estar en un todo de acuerdo con lo prescripto por él.

\section{Literatura citada}

Aguilar C., C. Ramírez, D. Rivera, K. Siu-Ting, J. Suárez \& C. Torres. 2010. Anfibios andinos del Perú fuera de áreas naturales protegidas: amenazas y estado de conservación. Revista Peruana de Biología 17 (1): 5-28. DOI: http://dx.doi.org/10.15381/ rpb.v17i1.46
Aguilar G. 2001. Access to genetic resources and protection of traditional knowledge in the territories of indigenous peoples. Environmental Science \& Policy 4 (4-5): 241-256. DOI: http://dx.doi.org/10.1016/S1462-9011(01)00028-4

Cabrera Medaglia J.A. 1994. Algunas Consideraciones sobre el Convenio de Diversidad Biológica. En: R. Salazar, J.A. Cabrera Medaglia y A. Lopéz Mora, eds. Biodiversidad, Políticas y Legislación a la Luz del Desarrollo Sostenible. Fundación AMBIO, San José: Pp. 61-83.

Caillaux Zazzali J., M. Ruiz Müller \& B. Tobin. 1999. El régimen andino de acceso a los recursos genéticos: lecciones y experiencias. Lima: Sociedad Peruana de Derecho Ambiental. 44 pp.

Chishakwe N. \& T.R. Young. 2003. Access to Genetic Resources, and Sharing the Benefits of their Use: International and Subregional Issues. Informe inédito. IUCN. 18 pp.

Comunidad Andina. 1996. Decisión N³ 391 que establece el Régimen Común sobre Acceso a los Recursos Genéticos. (en línea). http://www.wipo.int/wipolex/es/details.jsp?id=9446.

Comunidad Andina. 2000. Decisión Nº 486 que establece el Régimen Común de Propiedad Industrial. (en línea). http://www. wipo.int/edocs/lexdocs/laws/es/can/can012es.pdf

Declaración de Cancún de Países Mega-Diversos Afines. 2002. (en línea). https://www.cbd.int/doc/meetings/cop/cop-06/ information/cop-06-inf-33-es.pdf

Decreto Legislativo $N^{\circ}$ 1075. 2008. Decreto Legislativo que aprueba Disposiciones Complementarias a la Decisión 486 de la Comisión de la Comunidad Andina que establece el Régimen Común sobre Propiedad Industrial. 28 de junio de 2008. El Peruano Normas Legales: 375059- 375074

Decreto Supremo No 018-2015-MINAGRI. 2015. Aprueba el Reglamento para la Gestión Forestal. 30 de septiembre de 2015. El Peruano Normas Legales: 562528-562571

Decreto Supremo No 019-2015-MINAGRI. 2015. Aprueba Reglamento para la Gestión de Fauna Silvestre. 30 de septiembre de 2015. El Peruano Normas Legales: 562571-562607.

Decreto Supremo No 003-2009-MINAM. 2009. Eleva al rango de Decreto Supremo la Resolución Ministerial Nº 087-2008-MINAM y ratifican la aprobación del Reglamento de Acceso a los Recursos. 7 de febrero de 2009. El Peruano Normas Legales: 390445 - 390446 .

Decreto Supremo N ${ }^{\circ}$ 016-2015-MINAGRI. 2015. Aprueban nuevo Texto Único de Procedimientos Administrativos del Ministerio de Agricultura y Riego - MINAGRI. 19 de septiembre de 2015. El Peruano Normas Legales: 561793 - 561794.

FAO 2009. Tratado internacional sobre los recursos fitogenéticos para la alimentación y la agricultura. [en linea] $<\mathrm{ftp}: / / \mathrm{ftp}$.fao.org/ docrep/fao/011/i0510s/i0510s.pdf> (28/03/2016)

Glowka L. 2000. Bioprospecting, Alien Invasive Species, and Hydrothermal Vents: Three Emerging Legal Issues in the Conservation and Sustainable Use of Biodiversity. Tulane Environmental Law Journal 13 (2): 329-360.

Glowka L., F. Burhenne-Guilmin \& H. Synge. 1994. A Guide to the Convention on Biological Diversity. Gland and Cambridge: IUCN. $161 \mathrm{pp}$.

Godt C. 2009. Enforcement of Benefit- Sharing Duties in User Countries. In: E.C. Kamau and G. Winter, eds. Genetic Resources, Traditional Knowledge \& The Law. Solutions for Access and Benefit Sharing. Earthscan, London and Sterling. Pp. 419-438.

Kámiche Zegarra J. 2010. Resumen Nacional Ambiental Perú 2010. [s.l.]: United Nations Environment Programme. 100 pp.

Lago Candeira A. 2007. La Gobernanza Global del Medio Ambiente: ¿Se necesita una Organización Mundial? Tesis para optar al grado de Doctor. Facultad de Ciencias Jurídicas y Sociales. Universidad Rey Juan Carlos.

León B., N. Pitman \& J. Roque. 2006. Introducción a las plantas endémicas del Perú. Revista Peruana de Biología 13 (2): 9S-22S. DOI: http://dx.doi.org/10.15381/rpb.v13i2.1782

Lewis-Lettington R.J., M. Ruiz Müller, T.R. Young, K.A. Nnadozie, M. Halewood \& J. Cabrera Medaglia. 2006. Methodology for Developing Policies and Laws for Access to Genetic Resources and Benefit Sharing. Rome: International Plant Genetic Resources Institute. 35 pp. 
Ley $\mathrm{N}^{\circ}$ 29316. 2009. Modifica, incorpora y regula diversas disposiciones a fin de implementar el Acuerdo de Promoción Comercial suscrito entre el Perú y los Estados Unidos de América. 14 de enero de 2009. El Peruano Normas Legales: $388278-328283$.

MINAM (Ministerio del Ambiente) (a). 2010. (en línea). El Perú y el Cambio Climático. Segunda Comunicación Nacional del Perú a la Convención Marco de las Naciones Unidas sobre el Cambio Climático. 2010. 200 p. <http://unfccc.int/ resource/docs/natc/pernc2s.pdf>

MINAM (Ministerio del Ambiente) (b). 2010. (en línea) Cuarto Informe Nacional sobre la Aplicación del Convenio de Diversidad Biológica Años 2006-2009. https://www.cbd. $\mathrm{int} / \mathrm{doc} /$ world/pe/pe-nr-04-es.pdf

Naciones Unidas. 1992. Convenio sobre la Diversidad Biológica. [en línea] https://www.cbd.int/convention/text/

Naciones Unidas. 2002. Plan de Aplicación de las Decisiones de la Cumbre Mundial sobre el Desarrollo Sostenible. (en línea). http://www.un.org/spanish/esa/sustdev/pdf/WSSD_PlanImpl.pdf

Pacheco V., R. Cadenillas, E. Salas, C. Tello \& H. Zeballos. 2009. Diversidad y endemismo de los mamíferos del Perú. Revista Peruana de Biología 16 (1): 5-32. DOI: http://dx.doi. org/10.15381/rpb.v16il.111

Pastor Soplín S. 2004. Manejo de la Biotecnología Apropiada para Pequeños Productores: Estudio de Caso - Perú. Lima: REDBIO/FAO. 21 pp.

Plenge M. 2010. Lista de la Aves del Perú. Lima: SERNANP. 40 pp.

Convenio sobre la Diversidad Biológica. 2010. Protocolo de Nagoya sobre acceso a los recursos genéticos y participación justa y equitativa en los beneficios que se deriven de su utilización. (en línea). https://www.cbd.int/abs/text/default.shtml
Raustiala K. \& D.G. Victor. 1996. Biodiversity since Rio: The Future of the Convention of Biological Diversity. Environment 38 (4): 17-45. DOI: http://dx.doi.org/10.1080/00139157.19 96.9929252

Raustiala K. 1997. Domestic Institutions and International Regulatory Cooperation: Comparative Responses to the Convention on Biological Diversity. World Politics 49 (4): 482-509.

Resolución Ministerial 233-2013-MINAM. 2013. Disponen la publicación para fines de Consulta Pública de Proyecto de Decreto Supremo que aprueba el nuevo Reglamento de Acceso a Recursos Genéticos. 14 de agosto de 2013. El Peruano Normas Legales: 501090

Rosell M. 1997. Access to Genetic Resources: A Critical Approach to Decision 391 - Common Regimen on Access to Genetic Resources of the Commission of the Cartagena Accord. RECIEL 6 (3): 274-283.

Ruiz Müller M. 2008. Guía Explicativa de la Decisión 391 y una Propuesta Alternativa para Regular el Acceso a los Recursos Genéticos en la Sub-región Andina. Lima: Sociedad Peruana de Derecho Ambiental. 115 pp.

Silvestri L. 2014. La obligación de participación justa y equitativa en los beneficios derivados de la utilización de recursos genéticos establecida en el protocolo de Nagoya: juna oportunidad de desarrollo para países ricos en biodiversidad? Tesis para optar al grado de Doctor. Facultad de Ciencias Jurídicas y Sociales. Universidad Rey Juan Carlos. 
